\title{
Tensorial basis to the constancy of perceived object extent over variations of dynamic touch
}

\author{
CHRISTOPHER C. PAGANO, PAULA FITZPATRICK, and M. T. TURVEY \\ Center for the Ecological Study of Perception and Action \\ University of Connecticut, Storrs, Connecticut
}

\begin{abstract}
Subjects wielded occluded rods, with or without attached masses, and reported the distances reachable with their distal tips. Experiments 1-3 compared wielding about the wrist, the elbow, and the shoulder. Experiments 4 and 5 compared free wielding, using the whole arm, with wielding only about the wrist. The two comparisons, respectively, were of spatial and temporal variations in the rod's rotational inertia. Perceived extent was found to be constant in both comparisons. This constancy was tied to the inertia tensor $I_{i j}$ defined about a point that remains a fixed distance from the object during wielding-an invariant of the spatially and temporally dependent patterning of mechanical energy impressed upon the tissues of the body. Discussion focused on the reciprocal action and perception capabilities of multisegmented limbs, the tensorial relations in the neurobiology of dynamic touch, and the strategy of understanding perceptual constancy through invariants.
\end{abstract}

Gibson (1966) found that it is useful to differentiate among three types of touch-specifically, cutaneous, haptic, and dynamic-each of which can be considered as a subsystem of the haptic perceptual system. Cutaneous touch is the stimulation of skin and deeper tissues without movement of the joints. Haptic touch is the stimulation of the skin and deeper tissues with movement of the joints. Dynamic touch involves the stimulation of skin and other tissues in combination with muscular exertion. Although all three are the product of time-varying quantities of mechanical energy acting over the tissues of the body, dynamic touch is different from cutaneous and haptic touch in that the muscular exertion involved in producing a given distribution of mechanical energy is relevant to the properties being perceived. Gibson explained that dynamic touch "is a perceptual subsystem in its own right. More than any others, its perception is blended with performance, for the information comes from muscular effort. One can perceive the inertial properties of something only by wielding it" (1966, p. 128). The perception of object properties by wielding is a prominent example of dynamic touch. Most manual activities entail grasping and manipulating objects. In performing these acts, the hand is usually in contact with only part of the object, as when a cane is grasped by its end, a cup is grasped by its handle, an envelope is grasped by one of its corners, and so on. A person, without the benefit of seeing, can come to know about the spatial properties of objects, contacted tactually only in part, by the act of wielding them.

This work was supported by NSF Grant BNS-9011013, and an NSF predoctoral fellowship to $P$. F. The authors would like to thank James $R$. Lackner and Domina Eberle Spencer for comments made on earlier versions of this manuscript. Correspondence should be addressed to C. C. Pagano, Center for the Ecological Study of Perception and Action, University of Connecticut, 406 Babbidge Road, U-20, Storrs, CT 06269-1020.
Recent research has demonstrated that subjects are able to perceive the lengths of objects that they can wield but cannot see (Burton \& Turvey, 1990; Carello, Peck, \& Fitzpatrick, 1993; Fitzpatrick, Carello, \& Turvey, 1993; Pagano \& Turvey, 1993; Solomon \& Turvey, 1988; Solomon, Turvey, \& Burton, 1989a, 1989b). Additionally, wielding reveals something about an occluded object's shape (Burton, Turvey, \& Solomon, 1990), its orientation in the hand (Pagano \& Turvey, 1992; Turvey, Burton, Pagano, Solomon, \& Runeson, 1992), and the position of the hand's grasp relative to the object (Pagano, Kinsella-Shaw, Cassidy, \& Turvey, in press). In these experiments, subjects were mostly restricted to wielding objects by using motions about the wrist only. One would expect from everyday experience, however, that a perceived spatial property of a wielded object would be constant when that object was wielded about any one of several different joints. Unpublished observations by Solomon suggest that this is indeed the case (see Solomon et al., 1989b). Solomon asked subjects to perceive the distances reachable with rods wielded about the wrist or the wrist and elbow conjointly. Simple regressions predicting perceived length from actual length in the two conditions resulted in two linear and parallel functions, with intercepts separated by the length of the forearm. In the present series of experiments, the perceived length of a hand-held rod is examined for wielding about points of rotation located in the wrist, elbow, and shoulder, both singly and in combination.

Length, shape, orientation, and so on, are geometric properties. The haptic system, however, is stimulated by mechanical variables. The research mentioned above has identified the inertia tensor $I_{i j}$, a parameter comprising moments and products of inertia, as the relevant mechanical quantity to which perceiving by dynamic touch is tied. The dynamics of a wielded object held firmly in the hand, 
and thus the measurement of $I_{i j}$, have been referred to the rotation point in the wrist, the distance of the handheld object to that point being fixed. Experiments 1 and 2 are directed at the equivalence of perceived object length (or, more precisely, perceived distance reachable with an object) when that object is wielded about different joints, one at a time, where each joint corresponds to a point of rotation at a different distance from the object. Experiments 3-5 are directed at identifying the $I_{i j}$ underlying this perceptual equivalency.

\section{EXPERIMENTS 1 AND 2}

Grasp a stick firmly at one end and wield it with the eyes closed-first, with motions of the hand about the wrist; second, with motions of the forearm about the elbow while prohibiting motions of the hand about the wrist; and third, with motions of the whole arm about the shoulder while prohibiting separate motions about either the elbow or the wrist. One's impression of the length of the stick seems to be much the same in each case. From the understanding that object extent perceived through dynamic touch is related to the rotational inertia of the object, the apparent constancy of the stick's perceived length poses a puzzle. The resistance of an object to rotational acceleration is expressed as the sum of all the masses of the object multiplied by their distances squared from the point about which the object is rotated. As the distance from the rotation point increases, the object's resistance to a change in its rotational velocity increases as the square of the distance. Thus, a hand-held stick's rotational inertia is considerably greater for wielding about the elbow than about the wrist, and considerably greater, in turn, for wielding about the shoulder than about the elbow. If perceived extent is tied to rotational inertia, perceived extent should differ across the three conditions of wielding, rather than remain constant.

Experiments 1 and 2 were directed at determining whether an object is perceived to be of the same length when wielded about one of three different joints. In Experiment 1 , subjects were asked to perceive the distance reachable with a rod, which they wielded by using motions about one of three points of rotation that corresponded to the wrist, elbow, or shoulder, while keeping the irrelevant joints fixed voluntarily (see Figure 1). In Experiment 2 , subjects were asked to perceive the distance reachable with a rod by using motions about the wrist or elbow, with the wrist fixed on the elbow trials by an orthopedic splint.

\section{Method}

Subjects. Eight undergraduate students at the University of Connecticut participated in Experiment 1 as partial fulfillment of course requirements. Six subjects were men, and 2 were women. One subject was left-handed. Eight different men from the same population served as subjects in Experiment 2. All subjects in the second experiment were right-handed, although they were not recruited on this basis.
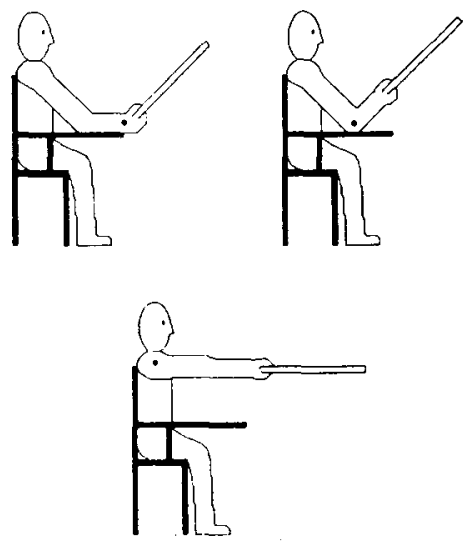

Figure 1. In Experiment 1, subjects wielded rods by using motions about the wrist, the elbow, or the shoulder. In Experiment 2, subjects wielded rods using motions about the wrist or the elbow.

Materials. The objects used in the experiments were four oak dowels $1.2 \mathrm{~cm}$ in diameter, with a density of $.671 \mathrm{~g} / \mathrm{cm}^{3}$. The four rods were $60,75,90$, and $105 \mathrm{~cm}$ long. An $82.5-\mathrm{cm}$ rod was used for instruction.

Apparatus. Figure 2 shows the experimental arrangement. The subject sat with the right forearm on or above a horizontal surface that was attached to the seat and occluded by a screen. Using a lever with the left hand, the subject was able to activate a motor that moved a white vertical surface toward or away from him/her. The subject could position the surface so that it was even with the edge of the horizontal surface, or up to $2.0 \mathrm{~m}$ away from it. From the experimenter's side of the screen, the position of the vertical surface could be read from a measuring tape, which was not visible to the subject.

Procedure. In each trial of both experiments, one of the four rods was placed in the subject's right hand, and the subject was asked to position the visible surface so that it corresponded to the distance that could be reached by the distal tip of the object. In all wielding conditions, the proximal tips of the rods were located at the edge of the horizontal surface, so that equal rod lengths corresponded to equal reachable distances under all modes of wielding. The subject was allowed to wield the rod and readjust the report surface for as long as was desired, until he/she was satisfied with his/her judgment. No movement of the rod relative to the hand was permitted in any conditions (the rod had to be grasped firmly and moved only by motions about the designated joints). No practice or feedback was given, and subjects were not told the number or lengths of rods used. The subjects were shown an $82.5-\mathrm{cm}$ rod as a sample of the objects they were to wield.

In Experiment 1, the subjects were asked to wield the object by using motions only about the wrist, elbow, or shoulder. They were instructed to keep the right forearm immobile against the horizontal surface during the wrist trials, and they were instructed to keep the right elbow immobile against the horizontal surface, and the right wrist voluntarily fixed, during the elbow trials. During the shoulder trials, they were asked to hold the entire right arm above the horizontal surface, and voluntarily to keep the right elbow and wrist fixed. The subjects experienced little to no trouble in complying with these instructions. However, if during any trial it appeared that the subject accidentally moved a joint that was supposed to be held fixed, the data from that trial were not recorded, and the trial was repeated at a later time. No subject required more than three such trials to be repeated. The three different points of rotation were used in random order, with the combination of four rod lengths and three points of rotation creating 12 different conditions. 

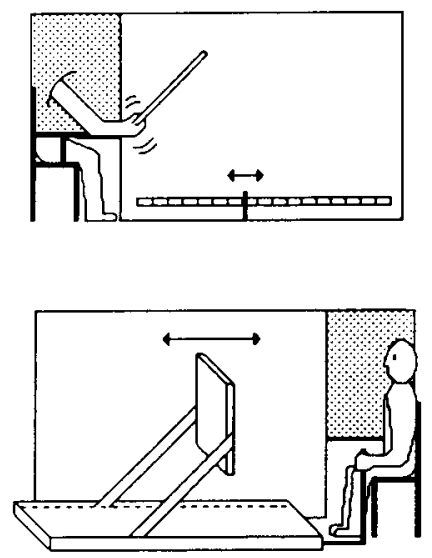

Figure 2. The apparatus used in the experiments. Top: The object to be wielded was occluded from the subject's view. Bottom: The motorized report apparatus, visible on the subject's left, was adjusted by a hand lever.

Each condition was presented three times, for a total of 36 trials per subject. During the experiment, the conditions were presented to the subject in random order, with all 12 conditions being run once before being repeated. The subjects were informed before each trial which point of rotation would be used in that trial.

In Experiment 2, subjects were asked to wield the object by using motions only about the wrist or the elbow. They were instructed to keep the right forearm immobile against the horizontal surface during the wrist trials, and the right elbow against the horizontal surface during the elbow trials. The right wrist was kept fixed during the elbow trials by an orthopedic wrist splint, which weighed $109 \mathrm{~g}$. The combination of four rod lengths and two points of rotation created eight different conditions. Each condition was presented four times for a total of 32 trials per subject. The wrist and elbow conditions were each split into two blocks of 8 trials for a total of four blocks. Half of the subjects used the wrist for the first and third blocks and the elbow for the second and fourth blocks; the other half used the elbow in the first and third blocks and the wrist in the second and fourth blocks. The blocks were created by randomly ordering the 16 trials for each point of rotation and then assigning the first 8 trials to the first block in which that point of rotation was used, and the second 8 trials to the second block in which that point of rotation was used. Thus it was possible for a particular block not to constitute a full set of rod lengths. The subjects could receive a rod in the third block, for example, that they did not receive in the first block, a fact that was carefully explained to each subject. This method was adopted so that subjects would not attempt to match the range of their responses in any one block to the range of responses in a previous block. The subjects were informed before each block of trials which point of rotation would be used during that block.

\section{Results}

Experiment 1. The perceived rod lengths averaged across subjects for the different points of rotation are presented in Table 1. Overall, the mean perceived length of the rods wielded about the wrist, elbow, and shoulder were $72.0,71.2$, and $74.3 \mathrm{~cm}$, respectively, and the mean perceived lengths of the $60-, 75-, 90-$, and $105-\mathrm{cm}$ rods were $49.5,60.3,80.2$, and $99.9 \mathrm{~cm}$, respectively. A $3 \times 4$ analysis of variance (ANOVA) with within-subject factors of rotation point and rod length confirmed a main effect of length $[F(3,21)=49.65, p<.0001]$, but not of rotation point $[F(2,14)=1.46, p>.10]$. The length $\times$ rotation point interaction was not significant $[F(6,42)=$ $1.01, p>.10$ ]. The ANOVA revealed that while subjects distinguished between the different rod lengths, perceived reachable distance remained invariant over different points of rotation.

Experiment 2. The perceived rod lengths averaged across subjects for the different points of rotation in Experiment 2 are presented in Table 2. Overall, the mean perceived length of the rods wielded about the wrist and elbow were 76.2 and $69.9 \mathrm{~cm}$, respectively, and the mean perceived lengths of the $60-, 75-, 90-$, and $105-\mathrm{cm}$ rods were $48.5,63.9,80.1$, and $99.6 \mathrm{~cm}$, respectively. A $2 \times 4$ ANOVA with within-subject factors of rotation point and rod length confirmed a main effect of length $[F(3,21)=$ $26.189, p<.0001]$, but not of rotation point $[F(1,7)=$ $2.49, p>.10$ l. The length $\times$ rotation point interaction was not significant $(F<1)$. As was the case in Experiment 1, the ANOVA for Experiment 2 revealed that while subjects distinguished between the different rod lengths, perceived reachable distance remained invariant over the different points of rotation. The relation of perceived reachable distance to actual reachable distance in Experiments 1 and 2 is depicted in Figure 3.

\section{EXPERIMENT 3}

As noted, previous research has isolated the parameter $I_{i j}$, the inertia tensor, as the relevant mechanical quantity to which perceiving by dynamic touch is tied. That research was focused primarily on the situation in which wielding occurred only about a point of rotation located in the wrist, so that the point of reference for $I_{i j}$ was taken to be in the wrist. Experiments 1 and 2 of the present article established the constancy of perceived object length when wielding occurred about one of three different points

Table 1

Mean Perceived Reachable Distance (in Centimeters) for the Different Points of Rotation Used in Experiment 1

\begin{tabular}{cccc}
\hline Acrual Length & Wrist & Elbow & Shoulder \\
\hline 60 & 55.0 & 51.9 & 53.3 \\
75 & 62.6 & 60.4 & 64.8 \\
90 & 78.8 & 79.8 & 85.8 \\
105 & 98.4 & 103.1 & 104.6 \\
\hline
\end{tabular}

Table 2

Mean Perceived Reachable Distance (in Centimeters) for the Different Points of Rotation Used in Experiment 2

\begin{tabular}{ccc}
\hline Actual Length & Wrist & Elbow \\
\hline 60 & 50.5 & 46.4 \\
75 & 66.3 & 61.4 \\
90 & 84.2 & 76.0 \\
105 & 103.6 & 95.6 \\
\hline
\end{tabular}




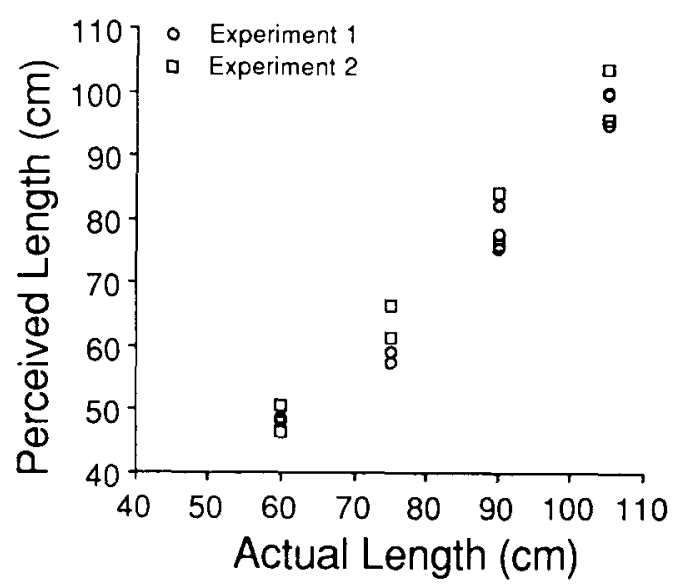

Figure 3. Perceived rod length as a function of actual rod length for Experiments 1 and 2.

of rotation, where each point was at a different distance from the object. Experiments 4 and 5 were directed at the perception of extent when rotational movements occurred at all joints simultaneously in comparison with when they occurred only at one, and they addressed the issue of what constituted the proper point of reference (if any) for $I_{i j}$ when all joints participated freely in the wielding. Before turning to Experiments 4 and 5, however, we develop the theoretical significance of $l_{i j}$ for dynamic touch and present an experiment-Experiment 3-that distinguished between two potential $I_{i j}$-based accounts of the constancy demonstrated in Experiments 1 and 2. As will become evident, $I_{i j}$ 's significance lies in the fact that it quantifies an invariant of the changing flux of mechanical energy available to the haptic system, and the hypothesized $I_{i j}$-based accounts of the constancy of perceived extent over different rotation points follow from this understanding.

\section{Gibson's (1966) Hypothesis and the Inertia Tensor}

Gibson hypothesized that "there is always some discoverable variable in stimulation-in the flowing array of energy at the sense organs of an animal-which determines the character of the perceptual process aroused by it" (1959, p. 457). More specifically, he hypothesized that the perception of object properties through dynamic touch, like perception in general, is possible through the pickup of invariants:

The mass of an object can be judged, in fact, by wielding it in any of a variety of ways, such as tossing and catching, or shaking it from side to side. One can only conclude that the judgment is based on information, not on the sensations. The stimulus information from wielding can only be an invariant of the changing flux of stimulation in the muscles and tendons, an exterospecific invariant in this play of forces. Whatever specifies the mass of the object presumably can be isolated from the change, and the wielding of the object has a function of separating off the permanent component from the changes. (Gibson, 1966, p. 127)
According to Gibson's hypothesis, the first step in the investigation of the perception of object properties through dynamic touch is to identify and measure mechanical parameters associated with the object that remain invariant during the act of wielding. One would then expect the perception of object properties to be tied to one such mechanical quantity, as opposed to some other quantity that changes during the act of wielding. That is, our task is to identify the invariant mechanical quantity to which the information underlying the perception of the object property is specific. Following this strategy, Solomon (1988; Solomon \& Turvey, 1988) isolated $l_{i j}$ as a parameter of special relevance to understanding the perceptual abilities of dynamic touch.

Rotational motions about a fixed point of the kind characteristic of wielding about a joint-as when the hand rotates around the wrist or the forearm around the elbow in the manipulation of an object-follow from $\mathbf{N}=\left(I_{i j} \cdot\right.$ $d \omega / d t)+\omega \times\left(I_{i j} \cdot \omega\right)$, where $\cdot$ is the scalar product and $X$ is the vector product (Goldstein, 1980). In the wielding of a given object, the torque $\mathbf{N}$, angular velocity $\omega$, and angular acceleration $d \omega / d t$, will vary from instant to instant. $I_{i j}$, in contrast, remains invariant. $I_{i j}$ is represented mathematically by a symmetric $3 \times 3$ matrix (Goldstein, 1980). The diagonal terms, the moments of inertia $\left(I_{x x}, I_{y y}, I_{z z}\right)$, quantify the object's rotational inertia with respect to three orthogonal axes $x y z$ centered at a point of reference $O$. The terms above $\left(I_{x y}, I_{x z}, I_{y z}\right)$ and below $\left(I_{y x}, I_{z x}, I_{z y}\right)$ the diagonal, the products of inertia, quantify the object's rotational inertia in directions perpendicular to the axial rotations and reflect the asymmetries of the object's mass distribution about the axes chosen. $I_{i j}$ is a symmetric tensor $\left(I_{x y}=I_{y x}\right)$ and thus contains six independent terms. In its diagonal form, $I_{i j}$ contains no off-diagonal terms and consists of three principal moments of inertia, or eigenvalues $\left(I_{1}, I_{2}\right.$, and $\left.I_{3}\right)$. The eigenvalues represent the rotational inertia of the object about each of three orthogonal axes centered at $O$, where the axes chosen are axes of symmetry of the object (termed principal axes of inertia, or eigenvectors). Thus the function for torque includes implicitly a number of constants or parameters independent of states and time. These parameters express the specific way in which the system is coupled to the forces imposed on it and thus convey upon the system its specific identity, independently of whatever radical changes occur in its states (Rosen, 1988).

The specific nature of the dependency of perception by dynamic touch on $I_{i j}$ is as follows: The maximum eigenvalue $I_{1}$ of $I_{i j}$ has been implicated as the relevant independent quantity in perceiving by wielding the lengths of rods or rod segments that cannot be seen, rather than other possibly relevant quantities such as torque, kinetic energy, muscular torsion, mass, center of mass, and center of oscillation (Burton \& Turvey, 1990; Carello et al., 1993; Solomon \& Turvey, 1988; Solomon et al., 1989a, 1989b). For example, for objects of identical mass but different rotational inertias, perceived length varied directly with 
rotational inertia (Solomon \& Turvey, 1988). $I_{1}$ has been found to predict the perceived lengths of irregularly shaped objects consisting of rods with attached branches of varying sizes whose forms and dimensions were unknown to the subjects (Pagano \& Turvey, 1993). Perceived length has also been found to be a function of both the maximum and the minimum eigenvalues $\left(I_{1}\right.$ and $\left.I_{3}\right)$ when subjects are tested with rods of varying composition or radii in a within-subject design (Fitzpatrick et al., 1993). Relatedly, perceived shape can be expressed as a function of the ratio of the maximum and minimum eigenvalues (Burton et al., 1990), and perceived object orientation in the hand has proven to be specific to the orientation of $I_{i j}$ 's eigenvectors (Pagano \& Turvey, 1992; Turvey et al., 1992), as is, apparently, the perceived position at which an object is grasped (Pagano et al., in press). These experiments provide confirmation of Gibson's (1966) hypothesis: The perceptions of the length and shape of an object are a function of $I_{i j}$, as are the perceptions of the hand-object spatial relations. The implication is that dynamic touch is tuned to the invariant parameters of the object's dynamics, rather than to the varying states (displacements, velocities) and torques.

As remarked, in almost all of the cited experiments, subjects were restricted to wielding objects by using motions only about the wrist, and thus $O$ was generally taken to be in the wrist. In Experiment 3, the perceived length of hand-held rods was examined for wielding about a point of rotation located in either the wrist $\left(O_{w}\right)$ or the elbow $\left(O_{e}\right)$. The objects used in the experiment consisted of three rods $1.2 \mathrm{~cm}$ in diameter and 60,75 , and $90 \mathrm{~cm}$ in length. A $60 \mathrm{~g}$ weight was added to each rod at $1 / 4$ of the rod's total length. The different rods could be placed in the subject's hand in one of two orientations, with the attached weight near to or far from the hand, corresponding to $1 / 4$ and $3 / 4$ of the the rod's total length, respectively. Thus, there were six different object conditions ( 3 rod lengths $\times 2$ weight positions). Three predictions are possible concerning the dependency of the perceived length of these objects on $I_{i j}$ when the objects are wielded about the two different $O$ s.

Prediction 1. The first prediction is that perceived length of the rods will scale to $I_{1}$ computed about the relevant $O$ in each trial. That is, perceived length will scale to $I_{1}$ computed about $O_{w}$ on the wrist trials, and to $I_{1}$ computed about $O_{e}$ on the elbow trials. Since the elbow is farther from the object than the wrist, $l_{1}$ computed about $O_{e}$ is greater than $I_{1}$ computed about $O_{w}$. Consequently, perceived length of objects wielded about the elbow should be much greater than that of objects wielded about the wrist. According to this first prediction, then, the perceived magnitude of a hand-held object is not constant when that object is wielded about different points of rotation. This prediction has already been ruled out by the results of Experiments 1 and 2.

Prediction 2. Fitzpatrick et al. (1993) investigated the perception of reachable distance with hand-held rods in cases where the rods varied either in radii or in composi- tion. $I_{1}$ is changed dramatically when rods of coincident lengths have different radii or compositions. Perceived reachable distance, however, changed much less dramatically and approximated the actual lengths of the rods more closely than would be predicted from $I_{1}$ alone. The basis for the invariance in perception over variance in $I_{1}$ is the fact that subjects were sensitive to $I_{i j}$ as a whole $\left(I_{1}, I_{2}\right.$, and $I_{3}$ ), rather than to any single component of $I_{i j}$. The second prediction, therefore, is that the perceived lengths of the rods would scale as a function of the eigenvalues of $I_{i j}$ in combination, where those eigenvalues are calculated with respect to the actual point of rotation $\left(O_{w}\right.$ or $O_{e}$ ) on a given trial.

Calculations of $I_{i j} \mathrm{~s}$ for the rods of Experiment 3 about the two different $O$ s reveal that for each rod length, even though the eigenvalues vary with both $O$ and weight condition (see Table 3 ), the magnitude of $.114\left(I_{1}^{32} I_{3}{ }^{37}\right)$ remains approximately the same. That is, the structure of the tensor is such that its components are correlated with rod length, independently of the point about which wielding occurs and the location of the added mass. Therefore, according to the second prediction, a given object will be perceived as having the same magnitude in both wrist and elbow trials, because $I_{i j}$ remains correlated with actual rod length in both cases. Furthermore, the perceived magnitude of the rod should remain invariant in conditions in which the added mass is located near or far with respect to the hand, because $I_{i j}$ computed about either $O$ remains correlated with actual rod length under both weight conditions. One further prediction is possible.

Prediction 3. The calculations of $l_{i j}$ 's diagonal and offdiagonal terms are made with respect to a rectangular coordinate system $O x y z$. Patently, there are indefinitely many sets of three perpendicular axes $x y z$ that can be anchored at $O$, and indefinitely many locations relative to the object at which to place $O$. For each choice of $O x y z$, the components of $l_{i j}$ will differ, but the nature in which the tensor specifies properties of the object does not change. This is a basic property of tensors (Lovett, 1989);

Table 3

Object Lengths (in Centimeters) and $I_{1}, I_{2}$, and $I_{3}\left(\mathrm{~g} \cdot \mathrm{cm}^{2} / 1000\right)$ for the Different Points of Rotation Used in Experiment 3

\begin{tabular}{ccrrr}
$\begin{array}{c}\text { Actual Rod } \\
\text { Length }\end{array}$ & $\begin{array}{c}\text { Weight } \\
\text { Condition }\end{array}$ & $I_{1}$ & \multicolumn{1}{c}{$I_{2}$} & $I_{3}$ \\
\cline { 1 - 5 } & \multicolumn{5}{c}{ Wrist $\left(O_{w}\right)$} \\
60 & $1 / 4$ & 76.32 & 74.84 & 1.57 \\
60 & $3 / 4$ & 193.72 & 184.32 & 0.70 \\
75 & $1 / 4$ & 125.49 & 123.91 & 1.68 \\
75 & $3 / 4$ & 294.24 & 293.57 & 0.76 \\
90 & $1 / 4$ & 221.16 & 219.32 & 1.94 \\
90 & $3 / 4$ & 357.09 & 356.78 & 0.40 \\
& & Elbow $\left(O_{e}\right)$ & & \\
60 & $1 / 4$ & 357.09 & 356.78 & 0.40 \\
60 & $3 / 4$ & 587.49 & 587.30 & 0.28 \\
75 & $1 / 4$ & 462.95 & 462.53 & 0.52 \\
75 & $3 / 4$ & 784.47 & 784.45 & 0.34 \\
90 & $1 / 4$ & 664.80 & 664.19 & 0.70 \\
90 & $3 / 4$ & 1091.41 & 1091.03 & 0.47 \\
\hline
\end{tabular}


inertia measured about one set of axes can be transformed to inertia measured about a different set of axes. In general, a tensor is a hypernumber-a matrix of independent numbers that expresses a physical state of affairs and that transforms in a particularly simple way (Moon \& Spencer, $1965,1986)$. Most significantly for our present purposes, it is a quantity that transforms in a given way with translation of coordinate systems. Different translations of $O$ result in different tensorial components (the numbers, termed merates, in the matrix change), but the manner in which the tensor transforms is such that it continues to define the object property that it quantifies. Thus, it is the case that we may translate $O$ from the actual point of rotation to some other location, and $I_{i j}$ computed about this new point will remain specific to the object properties of relevance to dynamic touch (see Pagano \& Turvey, 1992). In the present experiment, $I_{i j}$ computed about $O_{w}$, and $I_{i j}$ computed about $O_{e}$, can each be translated to a third $O$ to be taken as a common point of reference for both wielding conditions. For simplicity, this common $O$ will be located at the proximal end of the rod $\left(O_{p}\right)$, a point that approximates where the object is grasped and that remains constant over the two modes of wielding.

The third prediction holds that, regardless of the actual $O$, perception of object properties by dynamic touch will vary according to $I_{i j}$ computed about $O_{p}$. The $I_{i j} \mathrm{~s}$ computed about the actual point of rotation $\left(O_{w}\right.$ or $\left.O_{e}\right)$ in the different conditions of the experiment were transformed into $I_{i j} \mathrm{~s}$ about the coincident point $O_{p}$ (see Table 4). In short, the third prediction is that the perceived length of an object will vary as a function of $I_{i j}$ computed about $O_{p}$. Accordingly, a given object will be perceived as having the same magnitude in both wrist and elbow trials, because the $O$ about which $I_{i j}$ is defined is the same in both cases.

In sum, the three predictions for Experiment 3 were as follows: Although already eliminated by Experiments 1 and 2, Prediction 1 would be supported if perceived reachable distance did not remain constant over variation in either point of rotation and was found to be a function of $I_{1}$ computed about the relevant point of rotation $\left(O_{w}\right.$ or $O_{e}$ ) on a given trial. Prediction 2 would be supported if perceived reachable distance remained constant over variation in both point of rotation and weight condition and was found to be a function of $I_{i j}$ computed about the

Table 4

Object Lengths (in Centimeters) and $I_{1}$ and $I_{3}$ $\left(\mathrm{g} \cdot \mathrm{cm}^{2} / 1000\right)$ Computed About the Proximal End of the Rods $\left(O_{p}\right)$ for Experiment 3

\begin{tabular}{cccc}
\hline $\begin{array}{c}\text { Actual Rod } \\
\text { Length }\end{array}$ & $\begin{array}{c}\text { Weight } \\
\text { Condition }\end{array}$ & $\boldsymbol{I}_{\mathbf{1}}$ & $\boldsymbol{I}_{\mathbf{3}}$ \\
\hline 60 & $1 / 4$ & 69.95 & 0.96 \\
60 & $3 / 4$ & 177.95 & 0.96 \\
75 & $1 / 4$ & 118.09 & 0.97 \\
75 & $3 / 4$ & 286.84 & 0.97 \\
90 & $1 / 4$ & 211.33 & 1.00 \\
90 & $3 / 4$ & 454.33 & 1.00 \\
\hline
\end{tabular}

Note $-I_{1}=I_{2}$. relevant point of rotation $\left(O_{w}\right.$ or $\left.O_{e}\right)$ on a given trial. Prediction 3 would be supported if perceived reachable distance remained constant over variation in point of rotation and was found to be a function of $I_{i j}$ computed about $O_{p}$ in all trials.

\section{Method}

Subjects. Seven undergraduate students at the University of Connecticut participated in Experiment 3 as partial fulfillment of course requirements. Four subjects were men and 3 were women. One man and 3 women were left-handed; the remaining subjects were right-handed.

Materials. The objects used in the experiment consisted of three wood dowels $1.2 \mathrm{~cm}$ in diameter and 60,75 , and $90 \mathrm{~cm}$ in length. A $60-\mathrm{g}$ weight was added to each rod at $1 / 4$ of the rod's total length. An 82.5-cm-long rod was used for instruction.

Apparatus. This was the same as in Experiments 1 and 2.

Procedure. In half of the trials, one of the three rod lengths was placed in the subject's right hand so that the added weight was close to the hand; in the other half, it was placed with the added weight far from the hand. All other procedures were essentially the same as those used in Experiment 2. The subjects were asked to wield the object by using motions only about the wrist or elbow. They were instructed to keep the right forearm immobile against the horizontal surface during the wrist trials, and the right elbow against the horizontal surface during the elbow trials. The right wrist was kept fixed during the elbow trials by an orthopedic splint. The combination of three rod lengths, two weight conditions, and two points of rotation created 12 different conditions. Each condition was presented three times, for a total of 36 trials per subject. The wrist and elbow conditions were each split into two blocks of 9 trials for a total of four blocks. Half of the subjects used the wrist for the first and third blocks and the elbow for the second and fourth blocks; the other half used the elbow in the first and third blocks and the wrist in the second and fourth blocks. The subjects were not told the possible rod lengths used in the experiments, nor that the rods were weighted. No practice or feedback was given.

\section{Results and Discussion}

The perceived rod lengths averaged across subjects for the different points of rotation in Experiment 3 are presented in Table 5. Overall, the mean perceived reachable distances with the $60-, 75-$, and $90-\mathrm{cm}$ rods were 50.8 , 64.5 , and $77.0 \mathrm{~cm}$, respectively, and the mean perceived reachable distances with the weight at $1 / 4$ and at $3 / 4$ were 52.4 and $75.8 \mathrm{~cm}$, respectively. The mean perceived reachable distances with wielding about the wrist and elbow were 66.5 and $61.7 \mathrm{~cm}$, respectively. A $2 \times 2 \times 3$ ANOVA with within-subject factors of rotation point, weight condition, and rod length confirmed a main effect of each $[F(1,6)=9.301, p<.05 ; F(1,6)=129.089$, $p<.0001$; and $F(2,12)=48.709, p<.0001$, respectively]. There were no significant interactions. The main effect of length indicates that subjects distinguished reliably between the different rod lengths. The main effect of point of rotation, although marginal, indicated that subjects perceived rods wielded about the wrist to be longer than those wielded about the elbow. This effect of point of rotation is contrary to Prediction 1, by which rods wielded about the elbow were expected to be perceived to have greater magnitude than were those wielded about the wrist. The main effect of weight condition indicated that subjects reli- 
Table 5

Mean Perceived Reachable Distance (in Centimeters) for the Different Weight Conditions and

Points of Rotation Used in Experiment 3

\begin{tabular}{cccc}
\hline Actual Length & Weight Condition & Wrist & Elbow \\
\hline 60 & $1 / 4$ & 41.4 & 39.8 \\
60 & $3 / 4$ & 63.4 & 58.7 \\
75 & $1 / 4$ & 55.8 & 49.1 \\
75 & $3 / 4$ & 81.6 & 71.4 \\
90 & $1 / 4$ & 65.0 & 63.1 \\
90 & $3 / 4$ & 91.7 & 87.9 \\
\hline
\end{tabular}

ably perceived a greater reachable distance when the weights were located farther from the proximal end of the rod than when the weight was closer to the proximal end. This effect of weight is contrary to Prediction 2.

Simple regression of the mean perceived reachable distance $(\log P)$ on $\log I_{1}$ computed about the relevant point of rotation $\left(O_{w}\right.$ or $\left.O_{e}\right)$ for each condition resulted in an $r^{2}$ of .25. Multiple regression of $\log P$ on $\log I_{1}, \log I_{2}$, and $\log I_{3}$ computed about the relevant point of rotation $\left(O_{w}\right.$ or $\left.O_{e}\right)$ for each condition resulted in an $r^{2}$ of .58. Multiple regression of $\log P$ on the $\log I_{1}, \log I_{2}$, and $\log$ $I_{3}$ computed about $O_{p}$ resulted in an $r^{2}$ of .97 , with only $\log I_{1}$ significant after backward elimination $(p<.0001)$. The preceding results give evidence in favor of the third prediction and indicate that when the same object is wielded about one of several joints, the $I_{i j}$ s computed about the actual points of rotation can be translated to an $O$ common to all wielding conditions. The dependency of perceived reachable distance on $I_{1}$ computed about $O_{p}$ is depicted in Figure 4.

\section{EXPERIMENTS 4 AND 5}

The results of Experiment 3 indicate that perception by dynamic touch is tied to an invariant rendering of $I_{i j}$ when objects are wielded about different points of rotation. Typically, however, when an object is manipulated, one's motions are not restricted to a single joint, but rather occur about several joints freely. The purpose of Experiments 4 and 5 was to extend the findings of Experiment 3 to cases in which wielding occurred simultaneously about more than one point of rotation. In these experiments, subjects were asked to wield during half of the trials by using motions about the wrist only, and to use motions simultaneously about the wrist, elbow, and shoulder in the remaining trials. Thus, two modes of wielding, restricted and free wielding, were investigated in Experiments 4 and 5 (see Figure 5). The expected outcome followed from a consideration of the fact that a tensor is defined at a point. Consequently, when a number of points of rotation are active simultaneously for a given object, there will be defined an equal number of inertia tensors. In short, there is an inertia tensor field or $I_{i j}$ field. (In the present case, to each point in joint space [the joints of the arm], an $I_{i j}$ can be assigned.) If the distances of these points from the object are changing with time, the $I_{i j}$ field is a time-varying structure and the issue arises as to how an inconstant field might support the constant perception of object extent.

\section{An $I_{i j}$ Field Defined Over the Joints of the Arm}

When wielding occurs only about the wrist, the point of rotation for $I_{i j}$ can be taken to be in the wrist $\left(I_{i j} w r i s t\right)$. However, when wielding occurs freely about the wrist, elbow, and shoulder, each of the three joints can be taken as the point of reference for a different rendering of $I_{i j}$ ( $I_{i j}$ wrist, $I_{i j}$ elbow, and $I_{i j}$ shoulder, respectively). It is the case that two of these tensors, $I_{i j}$ elbow and $I_{i j}$ shoulder, will vary as a function of time. That is, because of movement about the wrist, the distance of the object from $O_{e}$ and, therefore, the mass distribution of the object about $O_{e}$, will change from instant to instant. Similarly, because of movement about the wrist and the elbow, the distance of the object from $O_{s}$ and, therefore, the mass distribution of the object about $O_{s}$ will change from instant to instant. The remaining tensor, $I_{i j}$ wrist, does not vary as a function of time-the distance of the object from $O_{w}$ and, therefore, the mass distribution of the object about $O_{w}$, does not change. In short, there is a field of $l_{i j} \mathrm{~s}$, only one of which is invariant with respect to time in both ex-

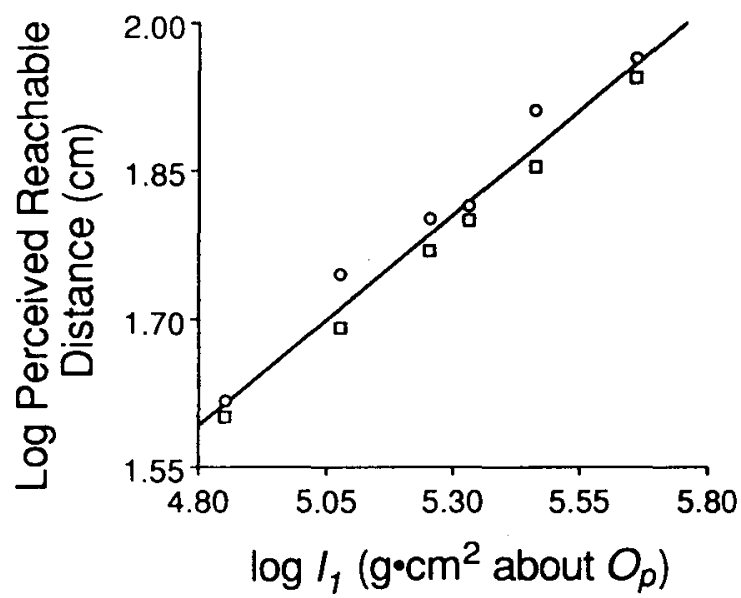

Figure 4. Perceived rod length as a function of the maximum eigenvalue of $I_{i j}$, computed about a point located at the proximal end of the rod for Experiment 3.
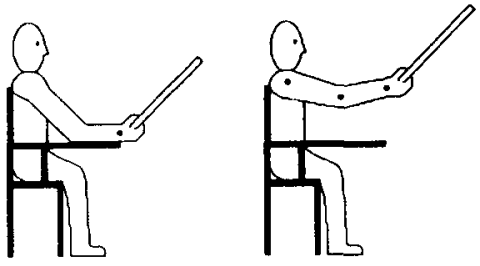

Figure 5. In Experiments 4 and 5, during half of the trials, subjects wielded by using motions about the wrist only, and used motions simultaneously about the wrist, elbow, and shoulder in the remaining trials. 
perimental conditions. By Gibson's (1966) hypothesis, one might expect that perceived object magnitude will vary as a function of $I_{i j}$ wrist in the free wielding condition, as well as in the restricted wielding condition, because in both cases $I_{i j}$ wrist quantifies an invariant of the changing flux of mechanical energy available to the haptic system. We would predict, therefore, that perception of object length will be the same under both modes of wielding-restricted and free-and will scale systematically to $I_{i j}$ wrist.

\section{Method \\ Subjects. Eight undergraduate students at the University of Con- necticut participated in Experiment 4 as partial fulfillment of course requirements. Three subjects were men and 5 were women. One woman was left-handed, and the other 7 subjects were right-handed. Eight different people ( 6 men, 2 women) from the same popula- tion served as subjects in Experiment 5 . All subjects in Experiment 5 were right-handed, although they were not recruited on this basis. \\ Materials. The objects from Experiment 3 were used in Experi- ment 4 . In Experiment 5, the subjects were given wooden rods 40 , 50 , or $60 \mathrm{~cm}$ long, with a $30-\mathrm{g}$ weight attached at a location $15 \mathrm{~cm}$ from one end. A 55-cm-long rod was used for instruction in Experiment 5. \\ Apparatus. This was the same as in the previous experiments. \\ Procedure. In each trial in both experiments, one of the rods was placed in the subject's right hand. The subject was asked to wield the rod by using motions only about the wrist, or to wield it freely about the wrist, elbow, and shoulder. In half of the trials, one of the three rod lengths was placed in the subject's hand so that the added weight was close to the hand; in the other half, it was placed with the added weight far from the hand. In both ex- periments, the two modes of wielding were given in random order, with the combination of three rod lengths, two weight conditions, and two modes of wielding creating 12 different conditions. Dur- ing the experiment, the conditions were presented to the subject in random order, with all 12 conditions being run once before be- ing repeated. Each condition was presented three times, for a total of 36 trials per subject. The subjects were informed before each trial which mode of wielding would be used in that trial.}

\section{Results and Discussion}

Experiment 4. Overall, the mean perceived reachable distances with the 60-, 75-, and 90-cm rods were 66.1, 80.7 , and $102.3 \mathrm{~cm}$, respectively, and the mean perceived reachable distances with the weight near and the weight far were 67.3 and $98.8 \mathrm{~cm}$, respectively. The mean perceived reachable distances in the restricted and free modes of wielding were 84.0 and $82.1 \mathrm{~cm}$, respectively. A $2 \times 2 \times 3$ ANOVA with within-subject factors of mode of wielding, weight condition, and rod length confirmed a main effect of weight condition $[F(1,7)=38.117, p<$ $.0001]$ and rod length $[F(2,14)=45.839, p<.0001]$, but not of mode of wielding $(F<1)$. The length $\times$ weight interaction was significant $[F(2,14)=5.926, p<$ $.05]$. The ANOVA reveals that while subjects distinguished between the different rod lengths, as well as the different weight conditions, perceived reachable distance was constant over the different modes of wielding.

Experiment 5. Overall, the mean perceived reachable distances with the $40-, 50-$, and $60-\mathrm{cm}$ rods were 42.5 ,
54.3 , and $64.4 \mathrm{~cm}$, respectively, and the mean perceived reachable distances with the weight near and the weight far were 46.9 and $60.5 \mathrm{~cm}$, respectively. The mean perceived reachable distances in the restricted and free modes of wielding were 53.6 and $53.9 \mathrm{~cm}$, respectively. A $2 \times 2 \times 3$ ANOVA with within-subject factors of mode of wielding, weight condition, and rod length confirmed main effects of weight condition $[F(1,7)=33.529, p<$ $.001]$ and rod length $[F(2,14)=45.604, p<.0001]$, but not of mode of wielding $(F<1)$. The length $\times$ weight interaction was significant $[F(2,14)=8.963, p<$ $.01]$. As was the case in Experiment 4, the ANOVA for Experiment 5 reveals that while subjects distinguished between the different rod lengths, as well as the different weight conditions, perceived reachable distance remained invariant over the different modes of wielding.

Simple regression predicting perceived rod length from actual rod length resulted in $r^{2} \mathrm{~s}=.45$ and .62 for Experiments 4 and 5, respectively (see Figure 6). Multiple regression predicting mean $\log$ perceived length from the maximum eigenvalue of $I_{i j}$ wrist $\left(\log I_{1}\right.$ wrist) resulted in an $r^{2}=.98$ in Experiment 4 and an $r^{2}=.98$ in Experiment 5 . Figure 7 depicts the single-valued dependence of $\log$ perceived length on $\log I_{1}$ wrist for the combined data of the two experiments.

The results of Experiments 4 and 5 indicate that perceiving the extent of an object by wielding is a function of an invariant rendering of $I_{i j}$, regardless of the actual number of degrees of freedom involved in the manipulation. It appears that the haptic system, operating over multiple or single degrees of freedom, follows Bernstein's (1967) principle of equal simplicity: If different structures execute a given function with the same degree of simplic-

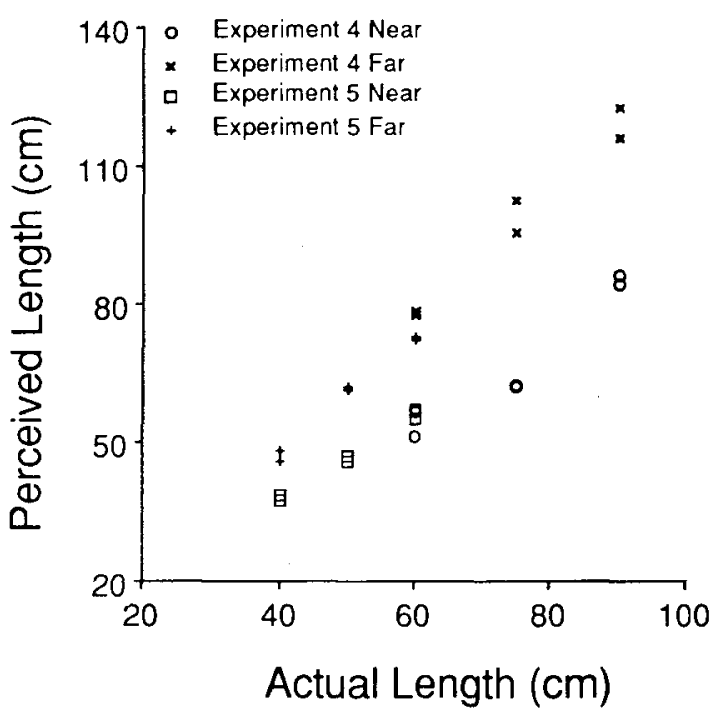

Figure 6. Perceived rod length as a function of actual rod length for the different weight conditions (mass attached to rod near to the hand vs. mass attached to rod far from the hand) used in Experiments 4 and 5 . 


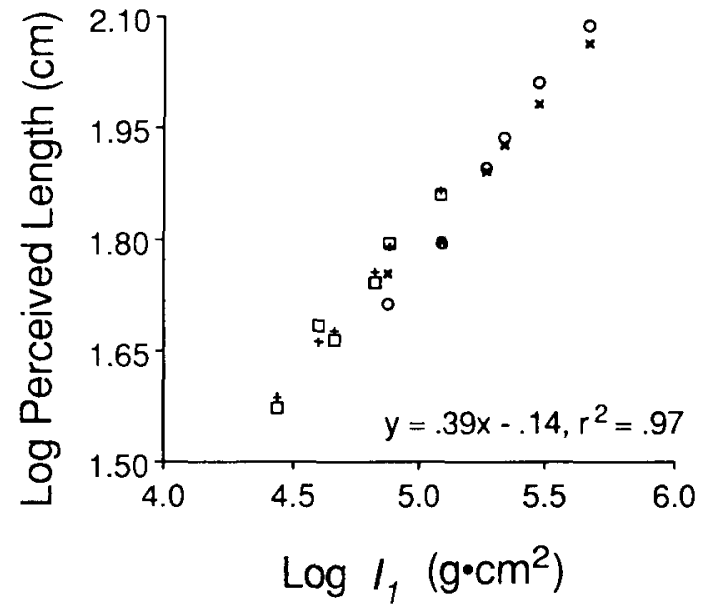

Figure 7. Perceived length is a single-valued function of the maximum eigenvalue of $\boldsymbol{I}_{i j}$ wrist. Circles indicate restricted wielding conditions, and Xs indicate free wielding conditions in Experiment 4. Squares indicate restricted wielding conditions, and crosses indicate free wielding conditions in Experiment 5.

ity, they may be considered to be organized in like fashion, abiding by the same principles of operation (see Carello, Fitzpatrick, Domaniewicz, Chan, \& Turvey, 1992). The present findings also demonstrate that, consonant with previous research (e.g., Solomon \& Turvey, 1988), object properties perceived by dynamic touch do not necessarily remain constant when the mass distribution of the object is altered (such as a rod of a given length with an added mass). The perception of properties of hand-held objects by dynamic touch seems to be dependent on the characteristics of an invariant rendering of $I_{i j}$ that are specific to the distribution of the object's mass about a relevant $O$.

The latter point echoes the conclusion favoring Prediction 3 in Experiment 3. A consideration of Experiment 3's results in the context of Experiments 4 and 5 suggests that when an object is firmly grasped so that it does not move relative to the hand in the course of wielding, the relevant $O$ is, in all likelihood, $O_{w}$. In Experiment 3 , the rotation point $O_{p}$ had been introduced in response to the need to identify a fixed distal rotation point, given that the candidate distal rotation point $O_{w}$ was ruled out, in theory, by the use of a splint that prohibited rotations about $O_{w}$. In retrospect, it seems more judicious to assume that, despite the constraining effect of the splint on the range of wrist deflections, the wielding of weighted rods incurred reactively small rotations about $O_{w}$ superimposed on the intentionally large rotations about $O_{e}$. That is to say, the relevant $O$ in Experiment 3 was $O_{w}$, and that highly restricted rotations about $O_{w}$ do not preclude a torques-tomotions dynamics about $O_{w}$ of sufficient magnitude to reveal the time-independent quantity $I_{i j}$ wrist and the timedependent quantity $I_{i j}$ elbow. Apparently, the haptic perceptual system is highly attuned to what is invariant about its own dynamics (see Solomon \& Turvey, 1988).

\section{GENERAL DISCUSSION}

The five experiments reported here demonstrate that the perception of the distance reachable with a rod grasped at an end, achieved by wielding the rod, is constant over (1) the different joints about which wielding can take place when those joints are taken singly, and (2) the contrast of wielding about one joint at a time versus wielding about all joints simultaneously. Importantly, the experiments have demonstrated that this constancy is tied to an invariant of the time-dependent patterning of mechanical energy impressed upon the tissues of the body. That invariant is the $I_{i j}$ associated with the given object about a point $O$ that remains a fixed distance from the object during wielding. For the commonplace case of wielding a firmly grasped object, the relevant point is $O_{w}$ and the governing $I_{i j}$ is $I_{i j}$ wrist. This conclusion dovetails with an empirical observation that was pivotal in establishing the moment-of-inertia hypothesis of extent perception by dynamic touch (Solomon, 1988; Solomon \& Turvey, 1988). According to the parallel axis theorem, an object's resistance to rotational acceleration is least when $O$ is at the object's center of mass (CM) and increases with deviations of $O$ from the CM. Solomon and Turvey (1988) and Solomon et al. (1989a) have found that the perceived length of a given rod wielded about $O_{w}$ depends on $O_{w}$ 's position relative to the rod's CM: The perceived length was least when the rod was grasped at its CM and most when grasped at an end. In sum, perceived reachable distance is a single-valued function of $I_{i j}$ wrist, a quantity that varies, by definition, with the coordinates of $O_{w}$ in the reference frame anchored at the wielded object's CM. In what follows, we consider several important implications of this conclusion.

\section{The Challenge of Haptically Perceiving with Multisegmented Limbs}

The universal biological shape seems to be cylindrical. Bodies of animals and plants are composed of cylindrical parts, approximately round or elliptical in cross-section with a readily identifiable longitudinal axis (Wainwright, 1988). The human arm consists of a number of cylindrical segments. When swung and waved, it behaves as a kinematic chain, with the torques and motions associated with any one cylindrical component affecting the torques and motions associated with the others, markedly or subtly (Bernstein, 1967). There are many skilled actions involving movements of the whole arm that require (1) a highly variegated dynamics of the kind made possible (actively and passively) by the arm's articulated cylinders, and (2) the continuous registration of the spatial magnitudes of a hand-held object to constrain the patterning, timing, and magnitude of the arm's muscular forces. Among the sports, lawn tennis, table tennis, badminton, hockey, baseball, and cricket immediately come to mind as examples of such activities. Because of the very obvious role of vision in the control and coordination of these acts, the role of dynamic touch is likely to go unnoticed. Without dy- 
namic touch, however, the scaling of muscular forces to the hand-held object's dimensions could not achieve the fluency and autonomy required by these skilled behaviors (e.g., Teasdale et al., 1992). Herein lies the rub. The two requirements of these skills identified above-(1) and (2)-would seem to be at odds with each other. In that dynamic touch is, by definition, tied to dynamics, requiring a highly variable movement dynamics would seem to contravene the requirement of an invariant spatial perception. The results of the present research reveal how the linked cylinder design resolves these apparently contradictory demands. Because the object in the hand remains at a fixed distance at all times from one particular distal joint-that which connects the cylinders constituting the hand to the forearm cylinder--there is always a feature of the implement's dynamics that remains constant regardless of how (necessarily) variable and flexible the arm-plus-implement's dynamics may happen to be. To reiterate, the constant is $I_{i j}$ wrist.

To use the arm as a perceptual instrument-a meter for the spatial properties of grasped objects-would be a problem of great complexity if the measures that the arm made were in terms of the time-dependent torques and motions. The complexity would be compounded if the time-varying $I_{i j}$ of the limb had to be factored into the computations. The present results, building on those of previous research, suggest that, contrary to the putatively complex calculations noted in the preceding sentence, the arm functions as a smart perceptual instrument (Bingham, 1988; Runeson, 1977; see also Carello et al., 1992; Solomon, 1988; Solomon \& Turvey, 1988). It capitalizes on that aspect of the complex play of mechanical forces on its tissues that does not change, and that connects physically with the spatial features of the object. Runeson (1977, p. 174) has suggested that the study of perception is the study of such smart perceptual instruments subdivided into "the search for the principles behind the function of the instruments, and the discovery of the physical realizations of these principles, i.e., how these instruments are actually built." He notes that the former would be the psychological part of the enterprise and the latter would be the physiological part. The physiological part is touched upon in the section that follows.

\section{Tensorial Relations in the Neurobiology of Dynamic Touch}

The neural basis for dynamic touch is to be found, primarily, in the muscle and tendon afferents. These afferents access both the somatosensory cortex and the motor cortex (Shepherd, 1988). When wielding occurs about all of an arm's joints simultaneously, the degree of muscular and tendinous afference is considerably richer in its space-time structure than when wielding takes place only about the wrist joint. It is also considerably larger in the number of degrees of freedom needed to characterize the active microstructural and muscular subsystems. Despite the contrast in complexity, Experiments 4 and 5 showed that the perceptual consequences of the two kinds of wield- ing are identical. Given that this perceptual sameness rests on a common basis in mechanical stimulation, namely, $I_{i j}$ wrist, a reasonable hypothesis is that it rests likewise on a common basis in afferentation. During both kinds of wielding, $I_{i j}$ wrist was the only invariant of the handheld object's rigid-body dynamics. Correspondingly, a particular relation among the muscular and tendinous afferents was the only invariant of the neural activity engendered by those dynamics. That is, in the two wielding conditions, perception was specific to one and the same invariant relation among the afferent nerves. How could this singular specificity be achieved? For the ultimate understanding of the haptic system's neurobiology, the preceding question poses at least two major problems. We provide sketches of the strategies with which each may be addressed.

How can a pattern of afference and $I_{i j}$ wrist function equivalently in constraining perception? The problem, at bottom, is to identify a conceptual structure with which the relations among very different quantities associated with one and the same physical situation can be understood. To anticipate, the sought-after conceptual structure is tensorial.

The rotational inertia of an object about a given point $O$ is a physical invariant-that is, a quantity independent of the coordinate systems at $O$ (e.g., rectangular, curvilinear, and those of both kinds produced through arbitrary rotations) by which it might be expressed. To reiterate, $I_{i j}$ wrist expresses the rotational inertia of the object relative to the point $O_{w}$. Wielding an object about $O_{w}$ incurs a time-dependent deformation pattern that is (1) constrained by the rigid arm-plus-rod dynamics, and (2) expressed in the intrinsic coordinate system defined by the muscles and tendons of the forearm (see Pellionisz \& Llinás, 1985). In concrete terms, at any given point in time, an array of numbers can be assigned to the states of the forearm's tissues. These numbers, referring as they do to the particular strain states, would be very different from the numbers by which the rigid body dynamics are described. Moreover, there would be more of them, given that the number of independent dimensions-muscles and tendons-to which quantities can be assigned exceeds the number of dimensions needed to formulate motions and torques in three-space. Nonetheless, a linkage must exist between the deformable body dynamics and the rigid body dynamics. The tensile states of muscles and tendons produce torques and motions of hand and object, and these in turn affect the tensile states of muscles and tendons. The two kinds of dynamics are co-implicative; there is a circular causality. Within this circular causality, an invariant deformation pattern $D_{k l}$ wrist (for simplicity, we assume that $D_{k l}$ is a second-order tensor) can be hypothesized to exist in correspondence with $I_{i j}$ wrist. $D_{k l}$ is $I_{i j}$ wrist in deformation quantities. The key property of a tensor is the transformation law-that is, the way its component quantities in one coordinate system are related to its component quantities in another coordinate system. The $n$ components of a tensor transform according to a definite 
law guaranteeing that the new components always determine the same tensor. ${ }^{1}$ For the general case, the precise form of this transformation law is a consequence of the physical or geometric meaning of the tensor (Borisenko $\&$ Tarapov, 1979). What relates $D_{k l}$ and $I_{i j}$ wrist, therefore, is a transformation law.

To continue this line of reasoning, it may likewise be hypothesized that the invariant rotational inertia about $O_{w}$ is also rendered as $A_{m n}$ wrist, the tensorial quantification of the invariant pattern of afferentation induced by $D_{k l}$ and expressed in the intrinsic coordinate system defined by the neural activity in the muscular and tendon afferents. There is, therefore, a rendering of the physical invariant of rotational inertia in at least three natural coordinate systems. These renderings, by definition, are not expressed identically and must differ in their details. But since they are all renderings of the same physical fact, they must be identical at the tensorial level of description. A tensor is a quantity that possesses a specified system of components in every coordinate system and that changes under a transformation of coordinates. Thus, $I_{i j}$ wrist, $D_{k l}$ and $A_{m n}$ wrist are renderings of the same fact in different coordinate systems.

Having been convinced that the available stimulation surrounding an organism has structure specific to its sources in the environment, Gibson (1966, p. 267) speculated that "If the invariants of this structure can be registered by a perceptual system, the constants of neural input will correspond to the constants of stimulus energy, although the one will not copy the other." The tensorial analysis of wielding expressed in the preceding paragraphs is consonant with this speculation of Gibson's and suggests how it might be treated rigorously.

How can there be selective attunement to $I_{i j}$ wrist? The problem is that the free wielding of Experiments 4 and 5 must have engendered large-scale, time-dependent activity in the afferents of the entire limb. Given that it did, how could the subject's perception have been selectively constrained by $l_{i j}$ wrist to the same degree that it was when wielding was restricted to the hand about the wrist? That is, how could $A_{m n}$ wrist be the only afference that mattered in free wielding? By the definition given, $A_{m n}$ wrist is a pattern of afferentation that remains unchanged as the afferentation of the limb changes. As the states of the afferent nerves transform in the act of free wielding, some aspect of their behavior is invariant. At issue is the disposition of the haptic system's neurobiology to respond preferentially to that which is left unchanged. It can be hypothesized that this disposition reflects a general principle (alluded to in the concluding remarks on Experiments 4 and 5): The neural substrate of the haptic perceptual system resonates to the invariants of its own dynamics.

\section{Concluding Remark: The Issue of Perceptual Constancy}

How one can have a constant perception of size, given the changing patterns of retinal stimulation accompanying variation in distance, has been a question at the forefront of visual perception theory. One time-honored hypothe- sis is that if one could register angle and could perceive object distance, object size could be calculated (Boring, 1942). Patently, if perceived size was always proportional to tangent [angle] $\times$ distance, size constancy would always be true. When the conditions on viewing deteriorate, as in the celebrated experiment of Holway and Boring (1941), perceived object size tends toward the size of the proximal stimulus. Specifically, if objects at different distances are made to project an image of identical size, then limiting vision to monocular viewing through a reduction screen (so that the surrounding surface layout is occluded and specifications of distance and spatial scale are minimized) renders the size perception of each object the same (Holway \& Boring, 1941). Because of such results, the perception has been labeled "size-at-a-distance," in contrast to simply "size" (e.g., Gibson, 1950; Koffka, 1935), and the time-honored hypothesis has been labeled the "size-distance invariance hypothesis" (e.g., Epstein, 1982). Furthermore, because the distance component is perceptual in the equation relating size perception to retinal angle and distance, it has been proposed that the timehonored hypothesis is continuous with the commonplace theoretical stance that perception is a ratiomorphic process. Constant size is perceived via an algorithm that couples percepts (e.g., Epstein, 1982).

The present research provides an example of the constancy of size perception in a nonvisual context. Expressed in the terms that have been developed primarily in the study of visual perception, the question posed in the introduction of Experiments 1-3 was: How could perception of the distal stimulus (a rod) remain the same when the proximal stimulus $\left(I_{i j}\right)$ was varied by increasing the distance of the distal stimulus from the point of observation $(O)$ ? In similar terms, the question posed to introduce Experiments 4-5 was: How could perception of the distal stimulus (a rod) remain the same when the proximal stimulus $\left(I_{i j}\right)$ varied in time? The strategy taken toward answering these questions was to seek in the dynamics of wielding a property that remained invariant over the spatial and temporal transformations. This strategy is continuous with Gibson's $(1959,1966)$ generalized informationperception specificity hypothesis: Perception is specific to information, and if perception is constant over spatially and temporally induced variations in stimulation, there must be a property of the stimulation that is likewise constant. The theoretical stance of the preceding rejects ratiomorphic processes as the basis of constancy and promotes, in their place, lawful relations between properties (see Barac-Cikoja \& Turvey, 1991; Turvey, 1992; Turvey et al., 1992). The isolation of $I_{1}$ of $I_{i j}$ wrist, as the constant property to which the constant perception of rod extent was related by a single-valued function, lends credence to this law-based perspective on the perceptual constancies.

\section{REFERENCES}

Barac-Cikoja, D., \& Turvey, M. T. (1991). Perceiving aperture size by striking. Journal of Experimental Psychology: Human Perception \& Performance, 17, 330-346. 
BERNSTEIN, N. A. (1967). The control and regulation of movements. London: Pergamon.

Bingham, G. P. (1988). Task-specific dynamics and the perceptual bottleneck. Human Movement Science, 7, 225-264.

BorING, E. G. (1942). Sensation and perception in the history of experimental psychology. New York: Appleton-Century-Crofts.

Borisenko, A. I., \& TARAPOV, I. E. (1979). Vector and tensor analysis with applications. New York: Dover.

Burton, G., \& Turvey, M. T. (1990). Perceiving the lengths of rods that are held but not wielded. Ecological Psychology, 2, 295-324.

Burton, G., Turvey, M. T., \& Solomon, H. Y. (1990). Can shape be perceived by dynamic touch? Perception \& Psychophysics, 48, 477-487.

Carello, C., Fitzpatrick, P., Domaniewicz, I., Chan, T. C., \& TURVEY, M. T. (1992). Effortful touch with minimal movement. Journal of Experimental Psychology: Human Perception \& Performance, 18, 290-302.

Carello, C., Peck, A., \& Fitzpatrick, P. (1993). Haptic and visual matches for haptically perceived extent are equivalent. Bulletin of the Psychonomic Society, 31, 13-15.

Epstein, W. (1982). Percept-percept coupling. Perception, 11, 75-83.

Fitzpatrick, P., Carello, C., \& Turvey, M. T. (1993). Eigenvalues of the inertia tensor and exteroception by the "muscular sense. "Manuscript submitted for publication.

GiBson, J. J. (1950). The perception of the visual world. Boston: Houghton Mifflin.

Gibson, J. J. (1959). Perception as a function of stimulation. In S. Koch (Ed.), Psychology: A study of science: Vol. I (pp. 456-501). New York: McGraw-Hill.

Girson, J. J. (1966). The senses considered as perceptual systems. Boston: Houghton Mifflin.

GoLDSTEIN, H. (1980). Classical mechanics. Reading, MA: AddisonWesley.

Holway, A. H., \& Boring, E. G. (1941). Determinants of apparent visual size with distance variant. American Journal of Psychology, 54, 21-37.

KoffKA, K. (1935). Principles of Gestalt psychology. New York: Harcourt, Brace.

Lovetr, D. R. (1989). Tensor properties of crystals. Philadelphia, PA: Adam Hilger.

Moon, P., SPencer, D. E. (1965). Vectors. Princeton, NJ: Van Nostrand.

MoOn, P., \& SPEncer, D. E. (1986). Theory of holors. Cambridge: Cambridge University Press.

Pagano, C. C., Kinsella-Shaw, J. M., Cassidy, P. E., \& Turvey, $M$. T. (in press). Role of the inertia tensor in haptically perceiving where an object is grasped. Journal of Experimental Psychology: Human Perception \& Performance.

Pagano, C. C., Turvey, M. T. (1992). Eigenvectors of the inertia tensor and perceiving the orientation of a hand-held object by dynamic touch. Perception \& Psychophysics, 52, 617-624.

Pagano, C. C., \& Turvey, M. T. (1993). Perceiving by dynamic touch the distances reachable with irregular objects. Ecological Psychology, 5, 125-151.

Pellionisz, A. (1986). Tensor network theory of the central nervous system and sensorimotor modeling. In G. Palm \& A. Aertsen (Eds.), Brain theory (pp. 121-146). Berlin: Springer-Verlag.

Pellionisz, A., \& Llinas, R. (1985). Tensor network theory of the metaorganization of functional geometries in the central nervous system. Neuroscience, 16, 245-273.

Rosen, R. (1988). Similarity and dissimilarity: A partial overview. Human Movement Science, 7, 131-153.

RUNESON, S. (1977). On the possibility of 'smart' perceptual mechanisms. Scandinavian Journal of Psychology, 18, 172-180.

ShEPHERD, G. M. (1988). Neurobiology (2nd ed.). New York: Oxford University Press.

Solomon, H. Y. (1988). Movement-produced invariants in haptic explorations: An example of a self-organizing, information-driven, intentional system. Human Movement Science, 7, 201-224.

Solomon, H. Y., \& TURVEY, M. T. (1988). Haptically perceiving the distances reachable with hand-held objects. Journal of Experimental Psychology: Human Perception \& Performance, 14, 404-427.

Solomon, H. Y., Turvey, M. T., \& Burton, G. (1989a). Gravitational and muscular variables in perceiving extent by wielding. Ecological Psychology, 1, 256-300.

Solomon, H. Y., Turvey, M. T., \& Burton, G. (1989b). Perceiving extents of rods by wielding: Haptic diagonalization and decomposition of the inertia tensor. Journal of Experimental Psychology: Human Perception \& Performance, 15, 58-68.

Teasdale, N., Bard, C., Fleury, M., Paillard, J., Forget, F., \& LAMARRE, Y. (1992, June). Bimanual interference in the absence of sensory information. Paper presented at the Human Frontier Science Program, Leuven, Belgium.

TURVEY, M. T. (1992). Ecological foundations of cognition: Invariants of perception and action. In H. L. Pick, P. van den Broek, \& D. C. Knill (Eds.), Cognition: Conceptual and methodological issues (pp. 85-117). Washington, DC: American Psychological Association.

Turvey, M. T., Burton, G., Pagano, C., Solomon, H. Y., \& RuneSON, S. (1992). Role of the inertia tensor in perceiving object orientation by dynamic touch. Journal of Experimental Psychology: Human Perception \& Performance, 18, 714-727.

WAINWRIGHT, S. A. (1988). Axis and circumference: The cylindrical shape of plants and animals. Cambridge, MA: Harvard University Press.

\section{NOTE}

1. Special issues are raised when the transformation is between coordinate systems that are dimensionally unequal, but suggestions for their resolution are to be found in the literature (e.g., Pellionisz, 1986; Pellionisz \& Llinás, 1985).

(Manuscript received July 24, 1992; revision accepted for publication January 7,1993 .) 